\title{
Solution of systems of partial differential equations by using properties of monogenic functions on commutative algebras
}

\author{
Tamila Kolomiets, Anatoliy Pogorui, \\ RAMÓN M. RODRÍGUEZ-DAGNino \\ (Presented by V. Ya. Gutlyanskii)
}

\begin{abstract}
In this paper some systems of differential equations with partial derivatives are studied by using the properties of Gâteaux differentiable functions on commutative algebras. The connection between solutions of systems of differential equations in partial derivatives and components of monogenic functions on corresponding commutative algebras is shown. We also give some examples of systems of differential equations with partial derivatives and find their solutions.
\end{abstract}

2000 MSC. Primary 35C99; Secondary 32W50.

Key words and phrases. PDE systems; Monogenic functions; Gâteaux derivative; commutative algebra.

\section{Introduction}

The study of partial differential equations (PDE) by using the properties of monogenic (Gâteaux differentiable) functions on commutative algebras sometimes makes it possible to effectively find solutions of these equations and investigate their characteristics. The best-known example of such an approach is the fact that the real and imaginary parts of a complex analytic function are harmonic functions, that is, solutions of the two-dimensional differential Laplace equation.

In [1-4] solutions of the multidimensional Laplace equation were studied. A generalization of this method to a wide class of partial differential equations with constant coefficients was implemented in [5]. The method consists in finding the commutative algebra associated with the differential equation and constructing monogenic functions on the corresponding subspace of this algebra. It is proved that in the case of a finite-dimensional algebra all the components of such a monogenic function are solutions of the corresponding equation. 
In [6] and [7] this method is applied to the study of one-dimensional distribution of the particles moving in Erlang-2 and Erlang-3 semi-Markov media.

In [8] the method is generalized to a PDE with linearly dependent variable coefficients.

In this paper the method of finding solutions by using monogenic functions is used to study the solutions of systems of partial differential equations with constant coefficients.

\section{Monogenic functions on commutative algebra}

Let $\mathbf{A}$ be a $n$-dimensional commutative algebra over a field $\mathbb{K}$ of characteristic 0 . Denote by $\vec{e}_{1}, \ldots, \vec{e}_{n}$ a basis of $\mathbf{A}$. Consider an $m$ dimensional subspace $\mathbf{B}$ of the algebra $\mathbf{A}$, where $m \leq n, m, n \in \mathbb{N}$ with the basis $\vec{e}_{1}, \vec{e}_{2}, \ldots, \vec{e}_{m}$. Suppose we have a function $\vec{f}: \mathbf{B} \rightarrow \mathbf{A}$ of the following form

$$
\vec{f}(\vec{x})=\sum_{k=1}^{n} u_{k}(\vec{x}) \vec{e}_{k},
$$

where $u_{k}(\vec{x})=u_{k}\left(x_{1}, x_{2}, \ldots, x_{m}\right)$ are $\mathbb{K}$-valued functions of $m$ variables $x_{i} \in \mathbb{K}$.

Thus,

$$
\text { B } \ni \vec{x}=\sum_{i=1}^{m} x_{i} \vec{e}_{i} \stackrel{\vec{f}}{\longrightarrow} \sum_{k=1}^{n} u_{k}\left(x_{1}, x_{2}, \ldots, x_{m}\right) \vec{e}_{k},
$$

such that $u_{k}: \mathbb{K}^{m} \rightarrow \mathbb{K}, k=1, \ldots, n$.

Definition 1. A function $\vec{f}$ is called differentiable at a point $\vec{x}_{0} \in \mathbf{B}$ if there exists a unique element $f^{\prime}\left(\vec{x}_{0}\right) \in \mathbf{A}$ such that for any $\vec{h} \in \mathbf{B}$

$$
f^{\prime}\left(\vec{x}_{0}\right) \vec{h}=\lim _{\mathbb{K} \ni \varepsilon \rightarrow 0} \frac{f\left(\vec{x}_{0}+\varepsilon \vec{h}\right)-f\left(\vec{x}_{0}\right)}{\varepsilon},
$$

where $f^{\prime}\left(\vec{x}_{0}\right) \vec{h}$ is the product of two elements $f^{\prime}\left(\vec{x}_{0}\right)$ and $\vec{h}$ of algebra $\mathbf{A}$.

Definition 2. A function $\vec{f}: \mathbf{B} \rightarrow \mathbf{A}$ is said to be monogenic if it is differentiable at every point $\vec{x} \in \mathbf{B}$.

Remark 1. It is easily seen that if $\mathbf{A}=\mathbf{B}=\mathbb{C}$ then a monogenic function is differentiable in the complex sense.

In papers $[5,8]$ the following two theorems were proved. 
Theorem 1. A function $\vec{f}(\vec{x})=\sum_{k=1}^{n} \vec{e}_{k} u_{k}(\vec{x})$ is differentiable at point $\vec{x}_{0}$ if and only if there exist partial derivatives $\frac{\partial \vec{f}\left(\vec{x}_{0}\right)}{\partial x_{i}}, i=1, \ldots, m$, which satisfy the following conditions

$$
\begin{array}{r}
u_{k}\left(x_{1}+\varepsilon h_{1}, x_{2}+\varepsilon h_{2}, \ldots, x_{m}+\varepsilon h_{m}\right)-u_{k}\left(x_{1}, x_{2}, \ldots, x_{m}\right) \\
=\varepsilon \sum_{i=1}^{m} \frac{\partial u_{k}}{\partial x_{i}} h_{i}+o(\varepsilon), \mathbb{K} \ni \varepsilon \rightarrow 0, \forall\left(h_{1}, h_{2}, \ldots, h_{m}\right) \in \mathbb{K}^{m}, \\
k=1,2, \ldots, n,
\end{array}
$$

and

$$
\vec{e}_{i} \frac{\partial \vec{f}\left(\vec{x}_{0}\right)}{\partial x_{j}}=\vec{e}_{j} \frac{\partial \vec{f}\left(\vec{x}_{0}\right)}{\partial x_{i}}, \quad i, j=1, \ldots, m
$$

Remark 2. The conditions $\vec{e}_{i} \frac{\partial \vec{f}\left(\vec{x}_{0}\right)}{\partial x_{j}}=\vec{e}_{j} \frac{\partial \vec{f}\left(\vec{x}_{0}\right)}{\partial x_{i}}, i, j=1, \ldots, m$ can be written in the following form

$$
\sum_{k=1}^{n} \frac{\partial u_{k}}{\partial x_{j}} \vec{e}_{i} \vec{e}_{k}=\sum_{k=1}^{n} \frac{\partial u_{k}}{\partial x_{i}} \vec{e}_{j} \vec{e}_{k} .
$$

Conditions (2.1) are called the generalized Cauchy-Riemann equations since when we have the special case $\mathbf{A}=\mathbf{B}=\mathbb{C}$ they are the Cauchy-Riemann equations.

For positive integers $r, m$ we introduce the following polynomial

$$
P\left(\xi_{1}, \xi_{2}, \ldots, \xi_{m}\right)=\sum_{i_{1}+i_{2}+\cdots+i_{m}=r} C_{i_{1}, i_{2}, \ldots, i_{m}}\left(x_{1}, x_{2}, \ldots, x_{m}\right) \xi_{1}^{i_{1}} \xi_{2}^{i_{2}} \ldots \xi_{m}^{i_{m}},
$$

where $C_{i_{1}, \ldots, i_{m}}\left(x_{1}, \ldots, x_{m}\right)$ are $\mathbb{K}$-valued continuous functions of the $m$ variables $x_{1}, \ldots, x_{m} \in \mathbb{K}$.

Now, let us consider the following differential equations

$$
P\left(\partial_{1}, \partial_{2}, \ldots, \partial_{m}\right)\left[u\left(x_{1}, x_{2}, \ldots, x_{m}\right)\right]=0,
$$

where $\partial_{k}=\frac{\partial}{\partial x_{k}}$.

Theorem 2. Let $P$ be a polynomial as in Eq. (2.2), and let a function $\vec{f}: \mathbf{B} \rightarrow \mathbf{A}$ be monogenic. In addition,

$$
\vec{f}(\vec{x})=\sum_{k=1}^{n} \vec{e}_{k} u_{k}(\vec{x}),
$$

where $\vec{e}_{1}, \vec{e}_{2}, \ldots, \vec{e}_{m}$ is a basis of the subspace $\mathbf{B}$ of the algebra $\mathbf{A}$ such that

$$
P\left(\vec{e}_{1}, \vec{e}_{2}, \ldots, \vec{e}_{m}\right)=0,
$$

then the functions $u_{k}(\vec{x}), k=1, \ldots, n$ are solutions of Eq. (2.3). 


\section{Differential functions proving solution to systems of PDEs.}

Consider the following system of PDEs

$$
\left\{\begin{array}{c}
D_{11} u_{1}(\vec{x})+D_{12} u_{2}(\vec{x})+\cdots+D_{1 N} u_{N}(\vec{x})=0 \\
D_{21} u_{1}(\vec{x})+D_{22} u_{2}(\vec{x})+\cdots+D_{2 N} u_{N}(\vec{x})=0 \\
\vdots \quad \vdots \\
D_{n 1} u_{1}(\vec{x})+D_{n 2} u_{2}(\vec{x})+\cdots+D_{N N} u_{N}(\vec{x})=0
\end{array}\right.
$$

where $D_{i j}$ are differential operators which satisfy commutative conditions $D_{i j} D_{k l}=D_{k l} D_{i j}, i, j, k, l=1,2, \ldots, N$.

Let us introduce the matrix of differential operators as follows

$$
D=\left(\begin{array}{cccc}
D_{11} & D_{12} & \cdots & D_{1 N} \\
D_{21} & D_{22} & \cdots & D_{2 N} \\
\vdots & \vdots & \ddots & \vdots \\
D_{N 1} & D_{N 2} & \cdots & D_{N N}
\end{array}\right)
$$

Denote by $\operatorname{det}(D)$ the determinant of $D$ and by $A_{i j}$ the algebraic complements of the matrix of elements of $D$, that is $\operatorname{det}(D)=D_{N 1} A_{N 1}+$ $D_{N 2} A_{N 2}+\cdots+D_{N N} A_{N N}$.

Theorem 3. Suppose $u_{i}(\vec{x}), i=1, \ldots, N$ is a solution of system (3.4), then for all $\lambda_{1}, \lambda_{2}, \ldots, \lambda_{N} \in \mathbb{K}$, we have

$$
\operatorname{det}(D)\left(\lambda_{1} u_{1}(\vec{x})+\lambda_{2} u_{2}(\vec{x})+\cdots+\lambda_{N} u_{N}(\vec{x})\right)=0 .
$$

Proof. The system of PDEs (3.4) can be represented in the vector-matrix form:

$$
D \vec{U}(\vec{x})=\overrightarrow{0}
$$

where $D$ is the matrix $(3.5)$, and $\vec{U}(\vec{x})=\left(\begin{array}{c}u_{1}(\vec{x}) \\ u_{2}(\vec{x}) \\ \vdots \\ u_{N}(\vec{x})\end{array}\right), \overrightarrow{0}=\left(\begin{array}{c}0 \\ 0 \\ \vdots \\ 0\end{array}\right)$.

Denote by $\tilde{D}=\left(A_{i j}\right)^{T}$ the transpose matrix composed of the complements $A_{i j}$.

Multiplying Eq. (3.7) by the matrix $\tilde{D}$, i.e., $(\tilde{D} D) \vec{U}=\tilde{D}(D \vec{U})=$ $\tilde{D} \overrightarrow{0}=\overrightarrow{0}$, we obtain 


$$
\left(\begin{array}{cccc}
\operatorname{det}(D) & 0 & \cdots & 0 \\
0 & \operatorname{det}(D) & \cdots & 0 \\
\vdots & \vdots & \ddots & \vdots \\
0 & 0 & \cdots & \operatorname{det}(D)
\end{array}\right)\left(\begin{array}{c}
u_{1}(\vec{x}) \\
u_{2}(\vec{x}) \\
\vdots \\
u_{N}(\vec{x})
\end{array}\right)=\left(\begin{array}{c}
0 \\
0 \\
\vdots \\
0
\end{array}\right)
$$

Hence,

$$
\left\{\begin{array}{c}
\operatorname{det}(D) u_{1}(\vec{x})=0 \\
\operatorname{det}(D) u_{2}(\vec{x})=0 \\
\vdots \\
\operatorname{det}(D) u_{N}(\vec{x})=0
\end{array}\right.
$$

or

$$
\operatorname{det}(D)\left(\sum_{i=1}^{N} \lambda_{i} u_{i}(\vec{x})\right)=0
$$

for all $\lambda_{1}, \lambda_{2}, \ldots, \lambda_{N} \in \mathbb{K}$.

Remark 3. It is easily seen that by solving Eq. (3.8) we obtain a linear combination of the system (3.4) with solutions $u_{i}(\vec{x}), i=1, \ldots, N$. Hence, if we have $m$ linear independent solutions $v_{i}(\vec{x}), i=1, \ldots, m$ of $\operatorname{det}(D) v(\vec{x})=0$ then we can look for a solution of the system (3.4) in the form of a linear combination as follows

$$
u_{i}(\vec{x})=c_{i 1} v_{1}(\vec{x})+c_{i 2} v_{2}(\vec{x})+\cdots+c_{i m} v_{m}(\vec{x}), \quad i=1,2, \ldots, m
$$

\section{Examples}

\subsection{Example 1.}

Consider the following system of PDEs

$$
\left\{\begin{array}{c}
\frac{\partial^{2}}{\partial x^{2}} u_{1}(x, y)+\frac{\partial^{3}}{\partial y^{3}} u_{2}(x, y)=0 \\
\frac{\partial}{\partial y} u_{1}(x, y)+\frac{\partial^{2}}{\partial x^{2}} u_{2}(x, y)=0
\end{array}\right.
$$

where $x, y \in \mathbb{R}$ and $u_{k}, k=1,2$ are real functions.

Let us write Eqs. (4.9) in the matrix form

$$
D \vec{U}(x, y)=\overrightarrow{0}
$$


where

$$
D=\left(\begin{array}{cc}
\frac{\partial^{2}}{\partial x^{2}} & \frac{\partial^{3}}{\partial y^{3}} \\
\frac{\partial}{\partial y} & \frac{\partial^{2}}{\partial x^{2}}
\end{array}\right), \quad \vec{U}=\left(\begin{array}{c}
u_{1}(x, y) \\
u_{2}(x, y)
\end{array}\right), \quad \overrightarrow{0}=\left(\begin{array}{c}
0 \\
0
\end{array}\right)
$$

In this case we have the following equation

$$
\operatorname{det}(D) v(x, y)=\left(\frac{\partial^{4}}{\partial x^{4}}-\frac{\partial^{4}}{\partial y^{4}}\right) v(x, y)=0 .
$$

Here we have the following corresponding polynomial $P\left(\xi_{1}, \xi_{2}\right)=$ $=\left(\xi_{1}^{2}\right)^{2}-\left(\xi_{2}^{2}\right)^{2}$. Then, we should find an algebra with basis $\vec{e}_{1}, \vec{e}_{2}$ satisfying

$$
P\left(\vec{e}_{1}, \vec{e}_{2}\right)=\left(\vec{e}_{1}^{2}\right)^{2}-\left(\vec{e}_{2}^{2}\right)^{2}=0 .
$$

It is easily seen that the basis $\{1, i\}$ of complex numbers satisfies Eq. (4.11), hence, we consider the case where $\mathbf{A}=\mathbf{B}=\mathbb{C}$.

Let us consider function $f(z)=e^{z}=e^{x}(\cos y+i \sin y)$. It is easily verified that $u_{1}(x, y)=C e^{x} \cos y-C e^{x} \sin y, u_{2}(x, y)=C e^{x} \cos y+$ $C e^{x} \sin y$, where $C \in \mathbb{R}$, is a solution of the system (4.9). It means that $c_{11}=-c_{12}=c_{21}=c_{22}=C$.

We should notice that for the system

$$
\left\{\begin{array}{c}
\frac{\partial}{\partial x} u_{1}(x, y)+\frac{\partial^{2}}{\partial y^{2}} u_{2}(x, y)=0 \\
\frac{\partial^{2}}{\partial y^{2}} u_{1}(x, y)+\frac{\partial^{3}}{\partial x^{3}} u_{2}(x, y)=0
\end{array}\right.
$$

In this case we also have $\operatorname{det}(D)=\left(\frac{\partial^{4}}{\partial x^{4}}-\frac{\partial^{4}}{\partial y^{4}}\right)$. Hence, we can consider the same function $f(z)=e^{x}(\cos y+i \sin y)$ to obtain a solution for (4.12).

It can be easily verified that $u_{1}(x, y)=C e^{x} \cos y+C e^{x} \sin y, u_{2}(x, y)=$ $u_{1}(x, y)$, is a solution of the system (4.12). This is also equivalent to say that $c_{11}=c_{22}=c_{12}=c_{21}=C$.

\subsection{Example 2.}

Let us solve the following system

$$
\left\{\begin{array}{c}
\frac{\partial}{\partial z} u_{1}(z, w)+\frac{\partial^{2}}{\partial w^{2}} u_{2}(z, w)=0 \\
\frac{\partial^{2}}{\partial w^{2}} u_{1}(z, w)+\frac{\partial}{\partial z} u_{2}(z, w)=0
\end{array}\right.
$$

where $z, w \in \mathbb{C}$ and $u_{k}, k=1,2$, are complex functions. 
Consider the matrix form of system (4.13)

$$
D \vec{U}=\overrightarrow{0}
$$

where

$$
D=\left(\begin{array}{cc}
\frac{\partial}{\partial z} & \frac{\partial^{2}}{\partial w^{2}} \\
\frac{\partial^{2}}{\partial w^{2}} & \frac{\partial}{\partial z}
\end{array}\right), \quad \vec{U}=\left(\begin{array}{c}
u_{1}(z, w) \\
u_{2}(z, w)
\end{array}\right), \quad \overrightarrow{0}=\left(\begin{array}{l}
0 \\
0
\end{array}\right) .
$$

For this case we have

$$
\operatorname{det}(D) v(z, w)=\left(\frac{\partial^{2}}{\partial z^{2}}-\frac{\partial^{4}}{\partial w^{4}}\right) v(z, w)=0
$$

In order to apply Theorem 2, instead of Equation (4.14), we solve the following equation

$$
\left(\frac{\partial^{4}}{\partial \omega^{2} \partial z^{2}}-\frac{\partial^{4}}{\partial w^{4}}\right) V(\omega, z, w)=0
$$

Let us find a solution of Eq. (4.15) in the form $V(\omega, z, w)=e^{\omega} v(z, w)$. It is easily seen that a function $v_{0}(z, w)$ is a solution of Eq. (4.14) if and only if the function $V(\omega, z, w)=e^{\omega} v_{0}(z, w)$ is a solution of Eq. (4.15).

The polynomial $P$ for Eq. (4.15) is as follows $P\left(\xi_{1}, \xi_{2}, \xi_{3}\right)=\xi_{1}^{2} \xi_{2}^{2}-\xi_{3}^{4}$. Hence, we should use a commutative algebra whose basis contains vectors $\vec{e}_{1}, \vec{e}_{2}, \vec{e}_{3}$ such that

$$
P\left(\vec{e}_{1}, \vec{e}_{2}, \vec{e}_{3}\right)=\vec{e}_{1}^{2} \vec{e}_{2}^{2}-\vec{e}_{3}^{4}=0 .
$$

For this case we can use bicomplex Segre numbers over a complex field as follows $\mathbf{A}=\left\{a_{0}+a_{1} j+a_{2} k+a_{3} f \mid a_{0}, a_{1}, a_{2}, a_{3} \in \mathbb{C}\right\}, j^{2}=k^{2}=$ $-1, f^{2}=1, j k=k j, j f=f j, k f=f k$, where $j, k, f$ commute with $i \in \mathbb{C}$.

Let us denote $i j=j i=p, i k=k i=q, i f=f i=r$. Then, $\mathbf{A}$ can be represented in the following form

$$
\mathbf{A}=\left\{a_{0}+a_{1} i+a_{2} j+a_{3} k+a_{4} f+a_{5} p+a_{6} q+a_{7} r \mid a_{i} \in \mathbb{R}\right\}
$$

with the following Cayley table

\begin{tabular}{|c|c|c|c|c|c|c|c|c|}
\hline & 1 & $i$ & $j$ & $k$ & $f$ & $p$ & $q$ & $r$ \\
\hline 1 & 1 & $i$ & $j$ & $k$ & $f$ & $p$ & $q$ & $r$ \\
\hline$i$ & $i$ & -1 & $p$ & $q$ & $r$ & $-j$ & $-k$ & $-f$ \\
\hline$j$ & $j$ & $p$ & -1 & $f$ & $-k$ & $-i$ & $r$ & $-q$ \\
\hline$k$ & $k$ & $q$ & $f$ & -1 & $-j$ & $r$ & $-i$ & $-p$ \\
\hline$f$ & $f$ & $r$ & $-k$ & $-j$ & 1 & $-q$ & $-p$ & $i$ \\
\hline$p$ & $p$ & $-j$ & $-i$ & $r$ & $-q$ & 1 & $-f$ & $k$ \\
\hline$q$ & $q$ & $-k$ & $r$ & $-i$ & $-p$ & $-f$ & 1 & $j$ \\
\hline$r$ & $r$ & $-f$ & $-q$ & $-p$ & $i$ & $k$ & $j$ & -1 \\
\hline
\end{tabular}


It is easily seen that $\mathbf{A}$ is associative and commutative. As the subspace $\mathbf{B}$ of the algebra $\mathbf{A}$ we consider

$$
\mathbf{B}=\left\{a_{0}+a_{1} f+a_{2} j \mid a_{i} \in \mathbb{C}\right\} .
$$

The basis $\{1, f, j\}$ of $\mathbf{B}$ satisfies Eq. (4.16).

Consider $\vec{f}: \mathbf{B} \rightarrow \mathbf{A}$ of the following form

$$
\begin{aligned}
\vec{f}(\omega, z, w) & =e^{\omega+f z+j w} \\
& =V_{1}(\omega, z, w)+V_{2}(\omega, z, w) j+V_{3}(\omega, z, w) k+V_{4}(\omega, z, w) f .
\end{aligned}
$$

According to Theorem 1 the function $\vec{f}$ is monogenic since

$$
\begin{gathered}
f \frac{\partial}{\partial \omega} \vec{f}(\omega, z, w)=\frac{\partial}{\partial z} \vec{f}(\omega, z, w)=f e^{\omega+f z+j w}, \\
j \frac{\partial}{\partial \omega} \vec{f}(\omega, z, w)=\frac{\partial}{\partial w} \vec{f}(\omega, z, w)=j e^{\omega+f z+j w}, \\
j \frac{\partial}{\partial z} \vec{f}(\omega, z, w)=f \frac{\partial}{\partial w} \vec{f}(\omega, z, w)=j f e^{\omega+f z+j w} .
\end{gathered}
$$

It follows from Theorem 2 that the components $V_{l}^{0}: \mathbb{C}^{3} \rightarrow \mathbb{C}$, $l=1,2,3,4$, are solutions of Eq. (4.15). Hence, the components of the function $\vec{f}(z, w)=e^{f z+j w}$ are solutions of Eq. (4.14). Let us write these components in more details

$$
\begin{aligned}
& \vec{f}(z, w)=e^{j w+f z}=e^{j w} \cdot e^{f z}=(\cos (w)+j \sin (w))(\cosh (z)+f \sinh (z)) \\
& =\cos (w) \cosh (z)+j \sin (w) \cosh (z)-k \sin (w) \sinh (z)+f \cos (w) \sinh (z) .
\end{aligned}
$$

Hence, we have the following solutions of Eq. (4.14)

$$
\left\{\begin{array}{cc}
v_{1}(z, w)=\cos (w) \cosh (z), & v_{2}(z, w)=\sin (w) \cosh (z), \\
v_{3}(z, w)=-\sin (w) \sinh (z), & v_{4}(z, w)=\cos (w) \sinh (z) .
\end{array}\right.
$$

Let us find a solution of system (4.13) in the following form

$$
\left\{\begin{array}{c}
u_{1}(z, w)=c_{11} \cos (w) \cosh (z)+c_{12} \sin (w) \cosh (z) \\
-c_{13} \sin (w) \sinh (z)+c_{14} \cos (w) \sinh (z), \\
u_{2}(z, w)=c_{21} \cos (w) \cosh (z)+c_{22} \sin (w) \cosh (z) \\
-c_{23} \sin (w) \sinh (z)+c_{24} \cos (w) \sinh (z) .
\end{array}\right.
$$

where $c_{i j} \in \mathbb{C}, i, j=1,2,3,4$. 
Substituting Eqs. (4.18) into Eq. (4.13), and simplifying these equations we obtain:

$$
\left\{\begin{array}{c}
\left(c_{11}-c_{24}\right) \cos (w) \sinh (z)+\left(c_{12}+c_{23}\right) \sin (w) \sinh (z) \\
-\left(c_{13}+c_{22}\right) \sin (w) \cosh (z)+\left(c_{14}-c_{21}\right) \cos (w) \cosh (z)=0 \\
\left(c_{11}-c_{24}\right) \cos (w) \cosh (z)+\left(c_{12}+c_{23}\right) \sin (w) \cosh (z) \\
-\left(c_{13}+c_{22}\right) \sin (w) \sinh (z)+\left(c_{14}-c_{21}\right) \cos (w) \sinh (z)=0
\end{array}\right.
$$

It follows from (4.19) that

$$
c_{11}=c_{24}, \quad c_{12}=-c_{23}, \quad c_{13}=-c_{22}, \quad c_{14}=c_{21}
$$

\section{Acknowledgments}

We thank the anonymous reviewer for his/her thorough review and highly appreciate the comments and suggestions.

\section{References}

[1] P. W. Ketchum, A complete solution of Laplace's equation by an infinite hypervariable // American Journal of Mathematics, 51 (1929), 179-188.

[2] C. Flaut, V. Shpakivskyi, An efficient method for solving equations in generalized quaternion and octonion algebras // Advances in Applied Clifford Algebras, 25 (2015), No. 2, 337-350.

[3] I. P. Melnychenko, Algebras of functionally invariant solutions of the threedimensional Laplace equation // Ukr. Math. Jour., 55 (2003), No. 9, 1284-1290 (in Russian).

[4] I. P. Melnychenko, S. A. Plaksa, Commutative algebras and spatial potential fields, Institute of Mathematics NAS of Ukraine, 230 pages, 2008 (in Russian).

[5] A. A. Pogorui , R. M. Rodríguez-Dagnino, M. Shapiro, Solutions for PDEs with constant coefficients and derivability of functions ranged in commutative algebras // Math. Meth. Appl. Sci., 37 (2014), No. 17, 2799-2810.

[6] A. Pogorui, The distribution of random evolutions in Erlang semi-Markov media // Theory of Stochastic Processes, 17 (2011), No. 1, 90-99.

[7] T. Kolomiiets, A. Pogorui, R. M. Rodríguez-Dagnino, The distribution of random motion with Erlang-3 sojourn times // Random Operators and Stochastic Equations, 23 (2015), No. 2, 67-83.

[8] A. A.Pogorui, R. M. Rodríguez-Dagnino, Solutions of some partial differential equations with variable coefficients by properties of monogenic functions // Ukr. Mat. Visn., 13 (2016), No. 1, 118-128; transl. in Journal of Mathematical Sciences, 220 (2017), No. 5, 624-632. 
Contact information

Tamila Kolomiiets Department of Mathematical Analysis, Zhytomyr State University, Zhytomyr, Ukraine E-Mail: tamila.kolomiiets@gmail.com

Anatoliy Pogorui

Department of Mathematical Analysis, Zhytomyr State University, Zhytomyr, Ukraine

E-Mail: pogor@zu.edu.ua

Ramón M. School of Engineering and Sciences, Rodríguez-Dagnino Tecnológico de Monterrey, Monterrey, México E-Mail: rmrodrig@itesm.mx 